\title{
Palinoestratigrafia do intervalo Alagoas da Bacia do Araripe, Nordeste do Brasil
}

\author{
Aristóteles de Moraes Rios-Netto ${ }^{*}$, Marília da Silva Pares Regali², Ismar de Souza Carvalho ${ }^{3}$, \\ Francisco Idalécio de Freitas ${ }^{4}$
}

\begin{abstract}
Resumo O conhecimento da história geológica da Bacia do Araripe traz contribuição efetiva ao entendimento da dinâmica evolutiva da geologia do Nordeste brasileiro. Apesar das inúmeras pesquisas realizadas nessa bacia quanto a seu rico conteúdo fossilífero - em especial no intervalo Alagoas -, poucos são os estudos dedicados à sua bioestratigrafia, um aspecto essencial quando se deseja entender a história evolutiva de uma bacia. Com o objetivo de fornecer uma moldura bioestratigráfica mais precisa para o Andar Alagoas da Bacia do Araripe, foram estudadas 167 amostras de testemunhos de 14 furos de sondagem perfurados na borda Leste dessa bacia. O intervalo, composto de arenitos, folhelhos, carbonatos e evaporitos, foi caracterizado litoestratigraficamente como pertencente às formações Rio da Batateira e Santana, enquanto as análises bioestratigráficas comprovaram a presença somente das subzonas P-270.2 e P-280.1, de Regali \& Santos (1999), na área estudada. A comparação dos resultados obtidos com dados gerados por outros autores permitiu o reconhecimento de intervalos correlatos nas bacias do Parnaíba, Grajaú, Potiguar e Sergipe.
\end{abstract}

Palavras-chave: Bacia do Araripe; Cretáceo Inferior; Bioestratigrafia; Palinologia; Andar Alagoas; Formação Rio da Batateira; Formação Santana.

\begin{abstract}
A pollen and spore based biostratigraphy of the Alagoas Stage (Lower Cretaceous) in the Araripe Basin, Northeastern Brazil. Any knowledge achieved about the geological history of the Araripe Basin contributes effectively for understanding the geology of the entire Northeastern Brazil. Besides the several researches concerning the rich fossiliferous content of that basin, specially of the Alagoas Stage, there are few studies focused on biostratigraphical aspects, which are especially important for understanding the geological evolution of a basin. Aiming at achieving a biostratigraphical framework to the Alagoas Stage in the Araripe Basin, palynological analyses were proceeded on 167 samples from 14 wells drilled at the Eastern portion of the Araripe Basin. The interval, composed of sandstones, microclastic sediments, limestones, and evaporites, was assigned to the Rio da Batateira and Santana Formations. The biostratigraphical analyses showed that only P-270.2 and P-280.1 subzones, from Regali \& Santos (1999), are present in the studied area. Comparison of these results with data from other authors allowed to recognize correlated intervals in the Parnaíba, Grajaú, Potiguar, and Sergipe basins.
\end{abstract}

Keywords: Araripe Basin; Lower Cretaceous; Biostratigraphy; Palynology; Alagoas Stage; Rio da Batateira Formation; Santana Formation.

\begin{abstract}
INTRODUÇÃO
A Bacia do Araripe está inserida no contexto do processo de abertura do Oceano Atlântico Sul e da divisão do paleocontinente Gondwana. A correlação com bacias sedimentares de seu entorno, as quais compartilham esse contexto evolutivo, como Parnaíba, Potiguar, Sergipe-Alagoas e Recôncavo-Tucano-Jatobá, concede relevância especial ao seu estudo, pois implica que o melhor conhecimento da história geológica da Bacia do Araripe traz contribuição efetiva ao entendimento da dinâmica evolutiva da geologia do Nordeste brasileiro.
\end{abstract}

O intervalo correspondente ao Andar Alagoas (formações Rio da Batateira e Santana) é o mais estudado de toda a coluna sedimentar da Bacia do Araripe, em parte devido ao riquíssimo conteúdo fossilífero, que se encontra entre os mais diversificados, abundantes e bem preservados em todo o mundo. No entanto, apesar dos inúmeros estudos paleontológicos, poucos trabalhos foram dedicados à bioestratigrafia da Bacia do Araripe. O presente estudo se propôs a contribuir com o conhecimento desse intervalo, por meio de um estudo bioestratigráfico baseado em

\footnotetext{
${ }^{1}$ Universidade Federal do Rio de Janeiro - UFRJ, Laboratório de Bioestratigrafia e Paleoecologia - LabMicro, Departamento de Geologia, Rio de Janeiro (RJ), Brasil. E-mail: rios.netto@geologia.ufrj.br

${ }^{2}$ Universidade Federal do Rio de Janeiro - UFRJ, Laboratório de Bioestratigrafia e Paleoecologia - LabMicro, Departamento de Geologia, Rio de Janeiro (RJ), Brasil. E-mail: regali.msp@gmail.com

${ }^{3}$ Universidade Federal do Rio de Janeiro - UFRJ, Centro de Ciências Matemáticas e da Natureza - CCMN, Instituto de Geociências IGEO, Departamento de Geologia, Rio de Janeiro (RJ), Brasil. E-mail: ismar@geologia.ufrj.br

${ }^{4}$ Universidade Regional do Cariri - URCA, Geopark Araripe, Crato (CE), Brasil. E-mail: idalecio@geoparkararipe.org

*Autor correspondente
} 
palinomorfos do Andar Alagoas na Bacia do Araripe. Os resultados obtidos também foram comparados com dados gerados por outros autores nas bacias do Parnaíba, Grajaú, Potiguar e Sergipe, permitindo o reconhecimento de intervalos correlatos nessas bacias.

MATERIAL E MÉTODOS Foram analisadas 167 amostras de sedimentos, procedentes de testemunhos de 14 furos de sondagem localizados na porção Nordeste da Bacia do Araripe (Fig. 1). Esses furos foram executados entre 1977 e 1978, durante a segunda etapa do "Projeto Santana", que foi desenvolvido pela Companhia de Pesquisa de Recursos Minerais (CPRM), por solicitação do Departamento Nacional da Produção Mineral (DNPM), com a finalidade de estudar a ocorrência e a viabilidade econômica de sulfetos de chumbo, zinco e cobre na região da Chapada do Araripe. A espessura da camada de sedimentos perfurada variou aproximadamente entre 56 e $224 \mathrm{~m}$. Os testemunhos estudados estão armazenados no Quarto Distrito do DNPM, em Recife, Pernambuco, que gentilmente os disponibilizou para o presente estudo. Para melhor compreensão da estratigrafia da bacia, foram realizadas, no âmbito do presente estudo, duas campanhas de campo, em julho de 2004 e em agosto de 2009.

O processamento das amostras palinológicas foi realizado pela equipe de técnicos do Laboratório de Palinofácies e Fácies Orgânicas (LAFO) do Departamento de Geologia do Instituto de Geociências da Universidade Federal do Rio de Janeiro, seguindo a metodologia proposta por Oliveira et al. (2004).

Optou-se por não se definir um biozoneamento local para o intervalo estudado; ao contrário, a opção escolhida foi buscar reconhecer na área estudada as unidades estratigráficas do biozoneamento de referência proposto por Regali et al. (1974) para o Brasil, considerando ainda a emenda de Beurlen \& Regali (1987) e a subdivisão em subzonas acrescentadas por Regali \& Santos (1999), para o Aptiano-Albiano.

\section{CONTEXTO GEOLÓGICO REGIONAL A}

Bacia do Araripe está localizada na região Nordeste do Brasil, cobrindo partes dos estados do Ceará, Pernambuco e Piauí. Em termos geomorfológicos, essa abrange as feições da Chapada do Araripe e do Vale do Cariri (Fig. 1). Sua área total é de aproximadamente $12.200 \mathrm{~km}^{2}$, com orientação geral LesteOeste, apresentando maior extensão nessa direção (ca. $250 \mathrm{~km})$ do que na Norte - Sul (ca. $68 \mathrm{~km})$. A bacia se insere na Zona Transversal da Província Estrutural Borborema, uma área marcada por diversos falhamentos do embasamento pré-cambriano, os quais mostram orientação predominante NordesteSudoeste e subordinada Nordeste-Sudoeste (Ponte \& Ponte-Filho 1996, Matos 1992). Em decorrência dessa herança estrutural, a arquitetura da Bacia do Araripe é fortemente caracterizada por horsts e grabens, estando compartimentada em duas sub-bacias, separadas pelo horst Dom Leme: a Sub-bacia de Feitoria, a Oeste dessa estrutura, e a Sub-bacia do Cariri, a Leste (Fig. 1). Os furos estudados se encontram na Sub-bacia do Cariri, sendo que a maioria deles foi realizado em uma calha estrutural de orientação aproximada Oeste-Noroeste - Leste-Sudeste; somente dois furos foram executados fora dessa calha sobre horsts (1-PS-09-CE e 1-PS-12-CE).

Diversas propostas estratigráficas têm sido apresentadas para a Bacia do Araripe, desde o estudo pioneiro de Small (1923) até a revisão mais recente de Assine (2007), dentre as quais podem ser destacadas algumas que representam etapas marcantes na evolução do conhecimento da estratigrafia dessa bacia, como: Beurlen (1962, 1963, 1971), Barros (1963), Gaspary \& Anjos (1964), Braun (1966), Silva-Santos \& Valença (1968), Mabesoone \& Tinoco (1973), Moraes et al. (1976), Silva (1986), Assine (1990, 1992), Ponte \& Appi (1990), Martill \& Wilby (1993) e Neumann \& Cabrera (1999). O intervalo estudado no presente trabalho corresponde ao Andar Alagoas (Coimbra et al. 2002) e abrange as formações Rio da Batateira (sensu Ponte \& Appi 1990) e Santana (sensu Beurlen 1962). Um total de 13, dos 14 furos de sondagem estudados, foi locado na encosta da Chapada do Araripe e não contém o topo da Formação Santana. Somente o furo 1-PS-12-CE foi perfurado no topo da Chapada, tendo atravessado a parte mais superior da coluna sedimentar da Bacia do Araripe, embora a testemunhagem tenha sido iniciada à profundidade de $106,3 \mathrm{~m}$, nível a partir do qual foram encontrados folhelhos. Esse furo foi, portanto, o único a testemunhar o topo daquela formação.

ESTUDOS ANTERIORES Apesar de a Bioestratigrafia não ser o objetivo principal do trabalho de Braun (1966), esse autor foi o primeiro a apresentar tal abordagem dos sedimentos da Bacia do Araripe, ao correlacionar a Formação Santana à sua Zona Z-7, de ostracodes, em um biozoneamento 


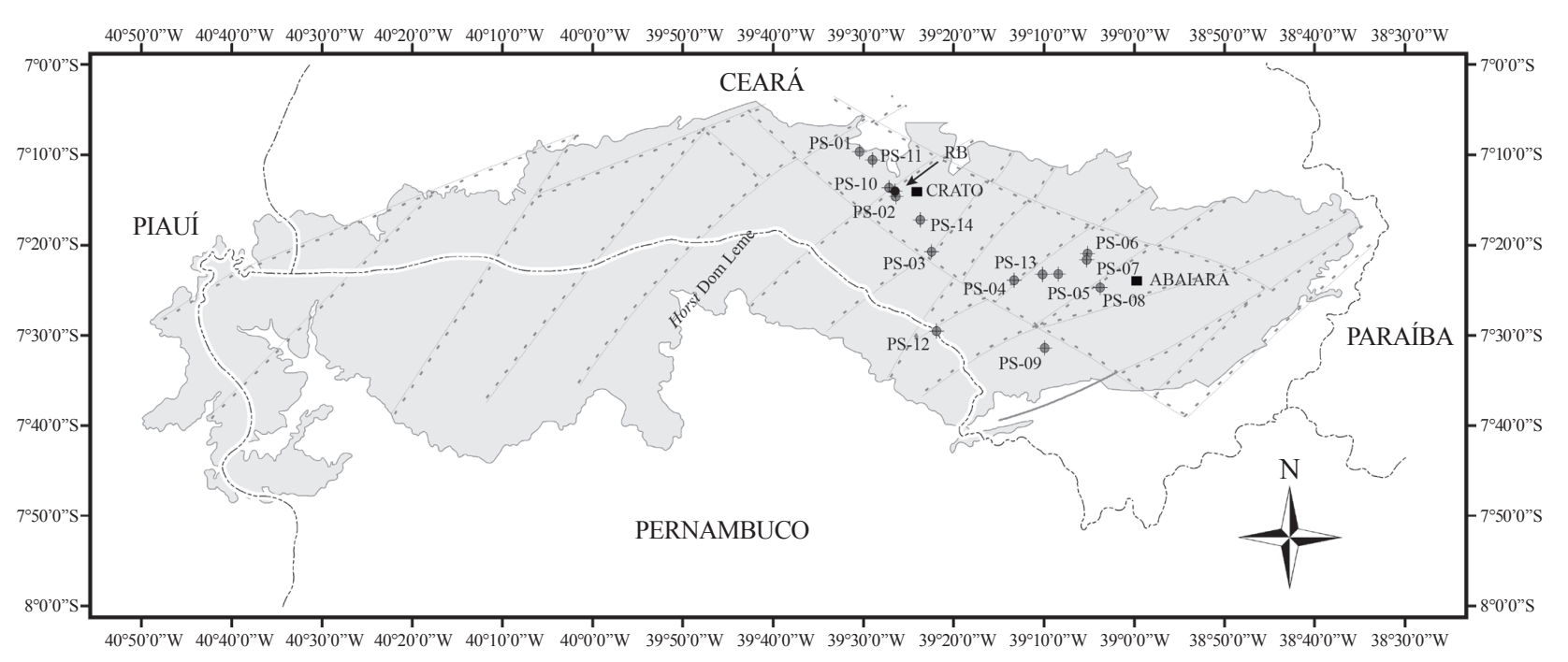

Figura 1 - Mapa da Bacia do Araripe mostrando as principais estruturas, o posicionamento dos furos de sondagem (PS-01 a PS-02) e do afloramento (RB) estudados, além das cidades mais próximas a esses pontos. A Sub-bacia do Cariri, a Leste do Horst Dom Leme, é fortemente marcada por uma calha estrutural de orientação aproximada Oeste-Noroeste - Leste-Sudeste (modificado de Ponte \& Ponte-Filho 1996).

regional que abarcava ainda as bacias de TucanoJatobá e Mirandiba.

Lima (1978) conduziu uma pesquisa palinológica detalhada sobre a Formação Santana, abordando Bioestratigrafia, Sistemática e aspectos paleoclimáticos, constituindo-se, até o momento, no mais amplo estudo desse tema na Bacia do Araripe. Lima (1978) dividiu a Formação Santana em quatro biozonas: Zona 1 (com as subzonas 1A a 1D), Zona 2, Zona 3 e Zona 4. Lima (1978) ressaltou a dificuldade que encontrou para correlacionar seu biozoneamento da Formação Santana com aquele proposto por Regali et al. (1974) para as bacias marginais, uma vez que não haviam sido encontradas as formas que aqueles autores usaram para caracterizar suas biozonas. No entanto, Lima (1978) considera que quase a totalidade da Formação Santana deveria ser correlacionada à Superzona Inaperturopollenites microclavatus (Superzona PC-30, atual Superzona P-300), devido à presença de uma forma que identificara como Galeacornea causea forma B, de idade albiana; para Lima (1978), somente a base da Formação Santana poderia ser correlacionável à Zona PC-28 (que corresponde à atual Zona P-280).

Mais tarde, Lima \& Perinotto $(1984,1985)$ assinalaram ao intervalo indiviso biozonas P-260 - P-270, de Regali et al. (1974), um nível de folhelhos pirobetuminosos posicionado entre arenitos sotopostos à Formação Santana. Uma amostra dessa mesma camada, coletada na localidade do Sítio Fundão (Crato,
Ceará) foi estudada por Regali (1990) e correlacionada com a Biozona P-270. Nesse mesmo trabalho, Regali (1990) estudou amostras dos membros Crato e Ipubi provenientes da borda oriental da Bacia do Araripe e as atribuiu à Biozona P-270. Regali (1990) também reavaliou os dados de Lima (1978), correlacionando-os à parte inferior da Zona P-280. Da integração desses dados, a autora inferiu uma discordância que corresponderia à parte basal da Zona P-280. Regali (1989) havia dividido essa biozona em quatro partes: basal, inferior, média e superior. Um aspecto interessante a ressaltar é que esta discordância foi inferida entre dados próprios de Regali (1990) e dados reavaliados de Lima (1978), o qual não havia observado a presença de Sergipea variverrucata em nenhuma amostra que estudou. Após Regali \& Santos (1999) subdividirem as zonas P-270 e P-280, em um estudo das bacias de Sergipe e Alagoas, Regali (2001) revisou novamente os dados palinológicos de Lima (1978) e concluiu que o intervalo estudado por esse autor corresponderia, na realidade, às subzonas P-280.1 e P-280.2. Regali (2001) também avaliou que a amostra estudada por Lima \& Perinotto (1984, $1985)$ e correlacionada por aqueles autores às biozonas P-260 - P-270 estaria restrita à Zona P-270.

Arai et al. (2000, 2001) e Coimbra et al. (2002) estudaram ostracodes e palinomorfos provenientes de amostras de afloramentos e também do poço 2-AP-1CE (Sub-bacia do Cariri). Esses trabalhos correlacionaram a Formação Rio da Batateira e o Membro Crato 
da Formação Santana à Zona P-270, de Regali et al. (1974), enquanto os membros Ipubi e Romualdo foram correlacionados a uma Zona Cicatricosisporites avnimelechi, não definida formalmente. Em relação ao zoneamento com ostracodes, ambas as formações foram correlacionadas com a Zona NRT-011, de Schaller (1969). Ostracodes também serviram de base para o trabalho de Syrio \& Rios-Netto (2002, 2004), que correlacionaram um nível correspondente ao topo da Formação Rio da Batateira ou base da Formação Santana a essa mesma Biozona NRT-011.

$\mathrm{O}$ trabalho bioestratigráfico mais recente sobre a Formação Santana foi apresentado por Portela (2008), que propôs a subdivisão dos sedimentos dessa unidade em quatro intervalos bioestratigráficos correlacionáveis, segundo a autora, às biozonas P-270 (“palinozonas A e B") e P-280 (“palinozonas C e D”), de Regali et al. (1974).

ANÁLISE E CORRELAÇÃO ESTRATIGRÁFICA DOS FUROS DE SONDAGEM Embora considerando também parâmetros litoestratigráficos, este trabalho é pioneiro na correlação bioestratigráfica dos furos de sondagem aqui estudados, uma vez que trabalhos anteriores enfocaram somente a correlação litoestratigráfica desse material (Scheid et al. 1978: todos os furos; Castro et al. 2006: 1-PS-11-CE, 1-PS12-CE, 1-PS-13-CE, 1-PS-14-CE; Moura-2007: 1-PS-07-CE, 1-PS-09-CE, 1-PS-10-CE, 1-PS-11CE, 1-PS-12-CE, 1-PS-13-CE; Paula-Freitas-2009: 1-PS-05-CE，1-PS-06-CE，1-PS-07-CE，1-PS-08CE, 1-PS-09-CE e 1- PS-13-CE; Paula-Freitas-2010: 1- PS-01-CE, 1-PS-05-CE, 1-PS-06-CE, 1-PS-07CE, 1-PS-08-CE, 1-PS-09-CE e 1- PS-13-CE).

Para cada um dos furos de sondagem, elaborouse um diagrama de distribuição dos palinomorfos presentes (os furos 1-PS-11-CE e 1-PS-12-CE, os mais completos, são apresentados nos Anexos 1 e 2). Quanto à caracterização bioestratigráfica, observase, em uma visão geral, relativamente pouca variação qualitativa tanto entre os furos analisados quanto ao longo de cada um deles. Dentre os táxons encontrados, destacam-se: Cardioangulina elongata, Pilosporites trichopapilosus, Klukisporites variegatus, $K$. reticulatus, K. foveolatus, Cicatricosisporites sp.1 Dino, C. microstriatus, C. avnimelechi,Lepitolepidites psarosus, Raistrikia obtusispina, Densoisporites perinatus, Crybelosporites brenneri, Caliallasporites spp., Antulsporites baculatus, Matonisporites silvai, Rugobivisiculites bahiasulensis, Uesuguipollenites callosus, Classopollis brasiliensis, C. alexi, C. spp., Gnetaceaepollenites spp., Equisetosporites spp., Sergipea variverrucata, Bennetitaepollenites regalii, Stellatopollis araripensis, S. spp., Tricolpites sagax, Afropollis operculatus, A. zonatus, Afropollis aff., A. jardinus, Dejaxpollenites microreticulatus. Essa associação microflorística aponta, de um modo geral, para o intervalo bioestratigráfico correspondente às subzonas P-270.2 e/ou P-280.1. No entanto, alguns fatores, como a profundidade relativamente pequena dos furos, o espaçamento irregular entre as amostras e a ausência ou ocorrência inconsistente de Sergipea variverrucata, dificultam a atribuição segura a uma ou a outra daquelas subzonas em muitos dos furos estudados. Somente nos furos 1-PS-11-CE e 1-PS-12-CE o quadro bioestratigráfico é mais claro, indicando a efetiva possibilidade de que o limite P-270.2/P-280.1 tenha sido alcançado. Nesses dois furos também foi possível observar uma leve variação vertical na microflora que se repete em alguns furos estudados, o que serve como parâmetro na correlação deles.

Nesse contexto, para se alcançar um posicionamento estratigráfico mais preciso dos furos de sondagem e também sua correlação, foi fundamental o estudo não apenas dos dados bioestratigráficos obtidos, mas também a integração desses dados com informações litoestratigráficas de Scheid et al. (1978), Moura (2007), Paula-Freitas (2009) e Ponte \& Appi (1990), além das observações de campo levantadas no presente trabalho. $\mathrm{O}$ reconhecimento do intervalo conhecido na literatura especializada como "camadas Batateira" (Biozona P-270, para Hashimoto et al., 1987, e Regali, 2001) nos diversos furos foi o passo inicial para o seu empilhamento. Esse reconhecimento se deu a partir da correlação de um perfil estratigráfico do rio Batateira, realizado com observações de duas campanhas de campo, em 2004 e 2009, com o furo 1-PS-02-CE (Fig. 2), perfurado próximo àquele riacho. A seguir, procedeu-se ao reconhecimento das demais unidades litoestratigráficas e sua correlação nos diversos furos. Uma vez realizada a correlação litoestratigráfica, verificou-se sua coerência com os resultados bioestratigráficos, incluindo as pequenas variações na microflora observadas verticalmente e entre os furos, o que permitiu refinar as interpretações bioestratigráficas e concluir que, na maioria dos furos estudados, somente a Subzona P-270.2 está presente; unicamente nos furos 1-PS-11-CE e 1-PS12-CE é possível, embora não possa ser totalmente assegurado, que a P-280.1 tenha sido alcançada. 
O resultado final das correlações lito- e bioestratigráficas é apresentado em três seções:

- $\quad \mathrm{A}-\mathrm{A}^{\prime}$ (Fig. 3), com direção NW - SE, que abrange a maior parte dos furos de sondagem: 1-PS-01-CE, 1-PS-11-CE, 1-PS-10-CE, 1-PS02-CE, 1-PS-14CE, 1-PS-03-CE, 1-PS-04-CE,

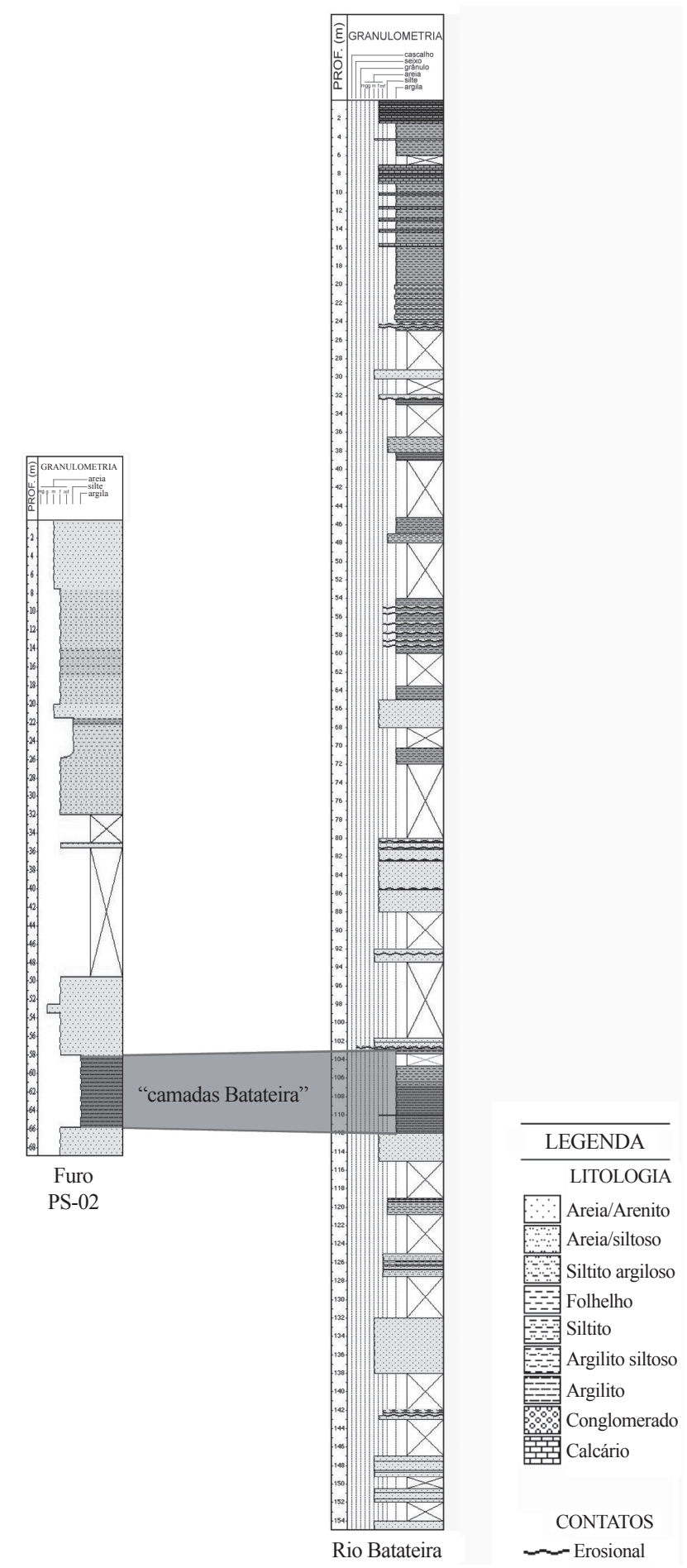

Figura 2 - Correlação entre o furo 1-PS-02-CE e o perfil do rio Batateira. Essa correlação permitiu a identificação do intervalo conhecido como "camadas Batateira" nos demais furos (perfil do furo 1-PS-02CE, modificado de Scheid et al. 1978).
1-PS-13-CE, 1-PS-05-CE e 1-PS-08-CE; essa seção é longitudinal em relação a uma calha que marca fortemente a arquitetura da Subbacia do Cariri (Fig. 1);

- B - B' (Fig. 4), com direção SW - NE, transversal à seção $\mathrm{A}-\mathrm{A}$ ': furos 1-PS-09-CE, 1-PS05-CE, 1-PS-07-CE e 1-PS-06-CE; é transversal em relação à calha citada (Fig. 1);

- $\quad$ C - C' (Fig. 5), com direção SSE - NNW, também transversal à seção $\mathrm{A}-\mathrm{A}^{\prime}$ : correlaciona os furos com maior amplitude bioestratigráfica: 1-PS-12-CE e 1-PS-11-CE; essa seção também é transversal à calha citada (Fig. 1).

As seções A-A' e B - B' foram escolhidas por seguirem as duas principais direções estruturais regionais (NW - SE e NE - SW). A seção C - C' correlaciona os dois furos de sondagem que alcançaram maior abrangência bioestratigráfica, dentre os analisados no presente estudo.

Além dessas seções de correlação, a Fig. 6 representa um perfil lito- e bioestratigráfico completo e idealizado da área estudada. É fruto do empilhamento estratigráfico dos perfis dos 14 furos de sondagem estudados, além da sucessão sedimentar exposta ao longo do rio Batateira (Crato, CE). Graficamente, esse perfil idealizado foi composto com partes dos perfis dos furos 1-PS-06-CE, 1-PS-10-CE e 1-PS-11-CE e do rio Batateira. Os furos 1-PS-10-CE e 1-PS-11-CE são complementares em termos estratigráficos e juntos já apresentam todas as unidades encontradas na área; eles formam a maior parte daquele perfil ideal. A porção do furo 1-PS-06-CE contendo as "camadas Batateira" foi acrescida na parte inferior do perfil, uma vez que é nesse furo que essas camadas ocorrem de modo mais completo. A parte mais inferior do perfil idealizado, abaixo das "camadas Batateira", foi tomada do perfil estratigráfico do rio Batateira por ser mais completa nessa localidade.

\section{CORRELAÇÃO COM BACIAS} SEDIMENTARES DO ENTORNO Como observado, somente a Subzona P-270.2 e, provavelmente, a parte inferior da Subzona P-280.1 estão presentes no intervalo estudado da Bacia do Araripe. Considerando-se a arquitetura estrutural da bacia, acredita-se que a Subzona P-280.2 possa estar presente na Sub-bacia de Feitoria (a qual não foi estudada neste trabalho), conforme advogado por Regali (2001). Assim, com base na associação palinológica encontrada no presente estudo, bem como naquelas 


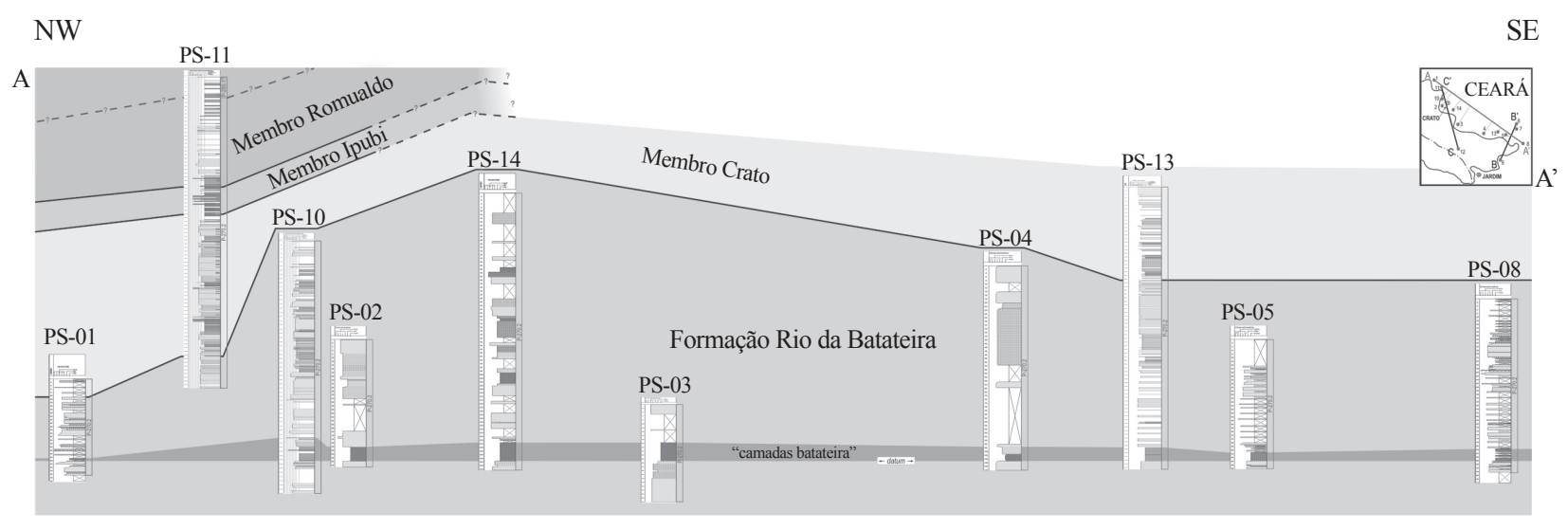

Figura 3 - Correlação lito e bioestratigráfica $N W-S E$ (seção $A-A$ '). Essa seção abrange a maior parte dos furos de sondagem estudados no presente trabalho, e sua direção coincide com a orientação de uma importante calha estrutural localizada na Sub-bacia do Cariri.

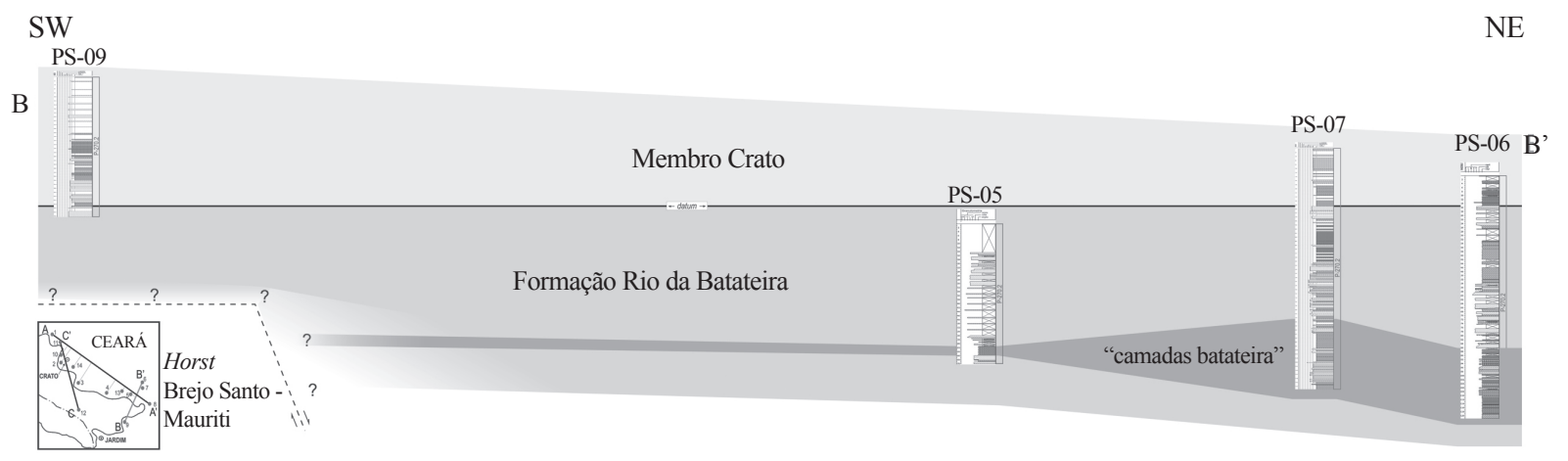

Figura 4 - Correlação lito e bioestratigráfica $S W-N E$ (seção $B-B^{\prime}$ ), transversal à seção $A-A^{\prime}$ '.

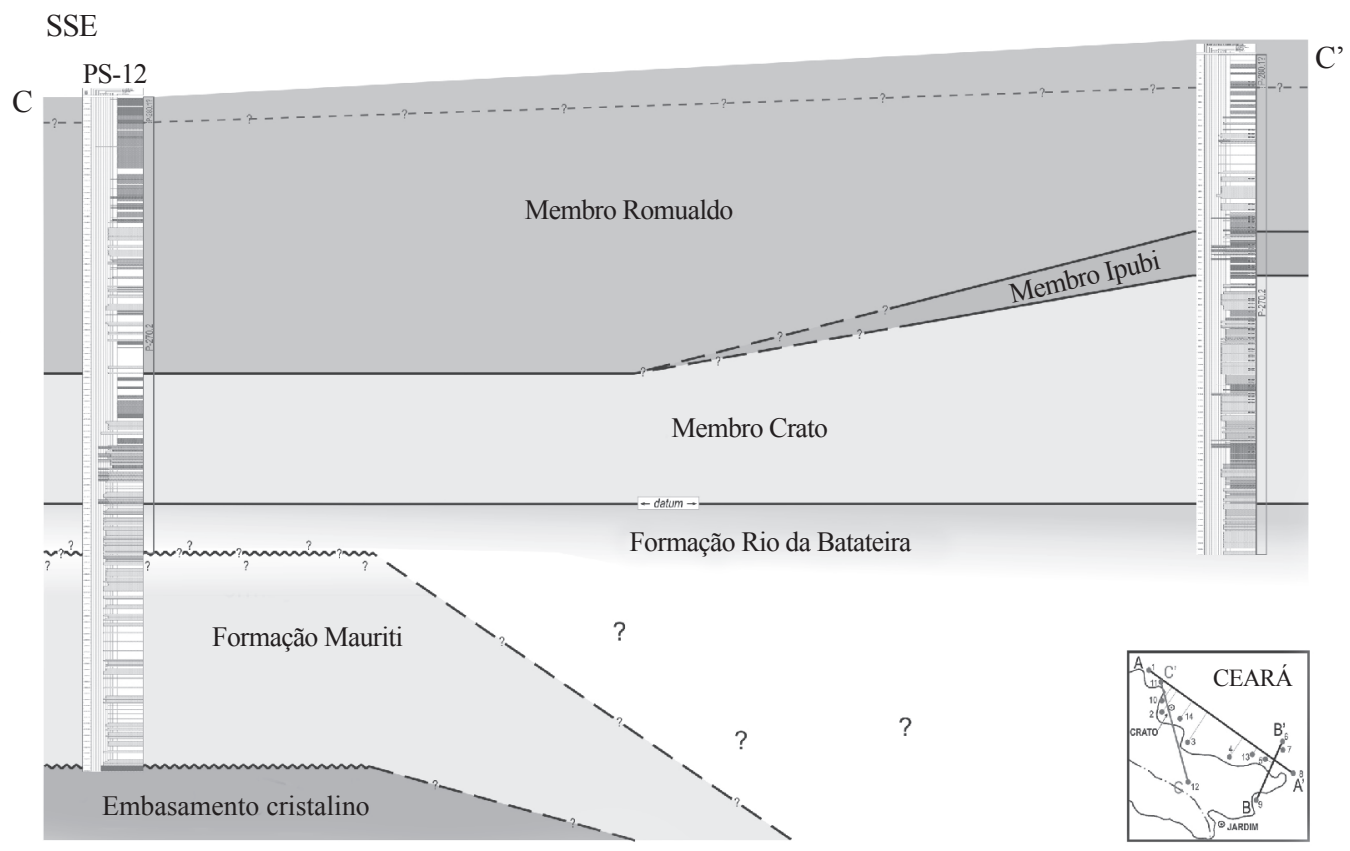

Figura 5 - Correlação lito e bioestratigráfica $N N W$ - SSE (seção C - C'). Essa seção correlaciona os dois furos de sondagem de maior amplitude estratigráfica dentre aqueles analisados neste estudo. 


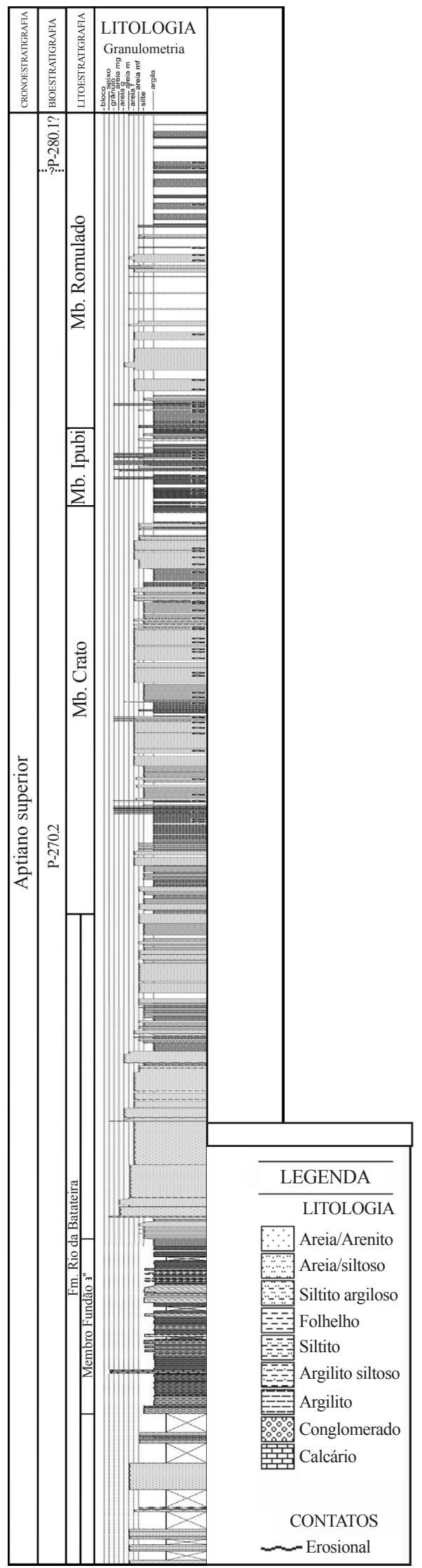

Figura 6 - Perfil lito e bioestratigráfico completo do intervalo estudado. Esse perfil idealizado foi composto com partes dos perfis 1-PS-06-CE, 1-PS10-CE e 1-PS-11-CE e rio Batateira (Fonte do perfil do furo 1-PS-06-CE: Paula-Freitas, 2009; fonte do perfil do furo 1-PS-11-CE: Moura 2007). citadas na bibliografia especializada, é possível afirmar que o intervalo formacional Rio da Batateira Santana é correlacionável aos seguintes intervalos das bacias do seu entorno:

- às biozonas $\mathrm{A}, \mathrm{B}$ e à parte inferior da Biozona C, que Dino (1992) reconheceu na Formação Alagamar (Bacia Potiguar): o topo da Biozona B é marcado pelo desaparecimento de Sergipea variverrucata e, portanto, é correlacionável com o topo da Subzona P-270.2 de Regali \& Santos (1999); a base de sua Biozona D é marcada pelo surgimento de Cretaceiporites polygonalis, indicando a correlação desse nível com a base da Subzona P-280.5 de Regali \& Santos (1999). Assim, somente a parte inferior de sua Biozona $\mathrm{C}$ pode ser ainda correlacionável ao intervalo formacional Rio da Batateira - Santana;

- às biozonas I a IV e à parte inferior da Biozona V, que Antonioli (2001) reconheceu na Formação Codó (Bacia do Parnaíba): o limite superior de sua Biozona IV é marcado pela extinção de Sergipea variverrucata, implicando na correlação desse nível com o topo da Subzona P-270.2 de Regali \& Santos (1999). As tabelas de distribuição estratigráfica dos palinomorfos nos poços 9-PCR-1-MA e 9-PIF-14-MA, estudados por Antonioli (2001), mostram que Cretaceiporites polygonalis surge na parte superior da Biozona V dessa autora, indicando que a partir desse nível o intervalo estudado seria correlacionável com a Subzona P-280.5, de Regali \& Santos (1999). Desse modo, somente a parte inferior da Biozona V de Antonioli (2001) pode ser correlacionável ao intervalo formacional Rio da Batateira - Santana;

- à parte do intervalo estudado por Carvalho (2001) nas formações Muribeca e Riachuelo (Bacia de Sergipe): no poço GTP-17-SE, esse autor reconheceu as subzonas P-270.2 e P-280.1, de Regali \& Santos (1999), em sedimentos das formações Riachuelo (membros Ibura e Oiterinhos) e Muribeca (Membro Angico); no poço GTP-24-SE, nas formações Riachuelo (membros Ibura e Oiterinhos) e Muribeca (Membro Taquari), foram encontradas as subzonas P-270.2, P-280.1 e P-280.2. Esses intervalos são, obviamente, correlatos 
do intervalo formacional Rio da Batateira - Santana;

- à "unidade inferior" e, talvez, à parte inferior da "unidade superior" do intervalo analisado por Rosseti et al. (2001) na Bacia de Grajaú: esses autores estudaram um intervalo da Bacia de Grajaú que dividiram em duas sucessões sedimentares. A tabela de distribuição estratigráfica dos palinomorfos que os autores apresentaram demonstra sua opinião de que a maior parte de sua "Unidade Inferior" é correlacionável à Biozona P-270 de Regali et al. (1974), enquanto a presença de Cretaceiporites polygonalis na amostra mais superior da "Unidade Superior" confirma sua correlação com um nível da Subzona P-280.5 ou mais alto.

CONCLUSÕES O intervalo estudado foi caracterizado litoestratigraficamente como pertencente às formações Rio da Batateira (partes média e superior) e Santana (membros Crato, Ipubi e Romualdo). Somente a Subzona P-270.2, de Regali \& Santos (1999), foi encontrada, com segurança, no intervalo estudado; o topo do intervalo provavelmente é correlacionável com a Subzona P-280.1, dos mesmos autores, embora a pequena amplitude dessa porção e o número reduzido de amostras não permitam assegurar a presença dessa subzona. Uma revisão dos dados palinológicos disponíveis na literatura especializada e a consideração da arquitetura estrutural da Bacia do Araripe sugerem que, além dessas unidades bioestratigráficas, somente a Subzona P-280.2, dos mesmos autores, deve estar presente no cretáceo inferior da bacia e deve ser encontrada principalmente em sua porção ocidental (Sub-bacia de Feitoria).

O intervalo formacional Rio da Batateira Santana é parcialmente correlacionável com os intervalos estudados por Dino (1992; Formação Alagamar, Bacia Potiguar), Antonioli (2001; Formação Codó, Bacia do Parnaíba), Carvalho (2001; parte superior da Formação Muribeca e parte inferior da Formação Riachuelo, Bacia de Sergipe) e Rosseti et al. (2001; parte da Formação Codó, Bacia de Grajaú).

AGRADECIMENTOS Este estudo contou com o apoio do Conselho Nacional de Desenvolvimento Científico e Tecnológico (CNPq), da Fundação Carlos Chagas Filho de Amparo à Pesquisa do Estado do Rio de Janeiro (FAPERJ), do Centro de Pesquisas Leopoldo Américo Miguez de Mello (CENPES)/PETROBRAS e da Fundação Coordenação de Projetos, Pesquisas e Estudos Tecnológicos (COPPETEC).

\section{Referências}

Antonioli L. 2001. Estudo Palino-cronoestratigráfico da Formação Codó - Cretáceo Inferior do Nordeste do Brasil. Tese de Doutoramento, Programa de Pósgraduação em Geologia, Instituto de Geociências, Universidade Federal do Rio de Janeiro, Rio de Janeiro, 303 p.

Arai M., Coimbra J.C., Silva-Telles A.C. 2000. Biostratigraphy of the Araripe Basin (Northeast Brazil, South America) based on microfossils. In: International Geological Congress, 31. Abstract Volume: arquivo E_ prgs_ArquivosPDF_G0202026.pdf.

Arai M., Coimbra J.C., Silva-Telles A.C. 2001. Síntese bioestratigráfica da bacia do Araripe (Nordeste do Brasil). In: Simpósio sobre a Bacia do Araripe e Bacias Interiores do Nordeste, 1 e 2. Comunicações, TIPROGRESSO, Crato, CE, Brasil, p.109-117, 122-124.

Assine M.L. 2007. Bacia do Araripe. Boletim de Geociências da Petrobras, 15(2):371-387.

Assine M.L. 1992. Análise estratigráfica da Bacia do Araripe, Nordeste do Brasil. Revista Brasileira de Geociências, 22(3):289-300.
Assine M.L. 1990. Sedimentação e Tectônica na Bacia do Araripe, Nordeste do Brasil. Dissertação de Mestrado, Programa de Pós-graduação em Geologia Regional, Instituto de Geociências e Ciências Exatas, Universidade Estadual Paulista, Rio Claro, 124 p.

Barros F.C. 1963. Sobre a sistemática da série Araripe. Engenharia, Mineração e Metalurgia, 37(218):32.

Beurlen K. 1971. As condições ecológicas e faciológicas da Formação Santana, na chapada do Araripe (Nordeste do Brasil). Anais da Academia Brasileira de Ciências, 43(suplemento):411-415.

Beurlen K. 1963. Geologia e estratigrafia da Chapada do Araripe. In: Congresso Nacional de Geologia, 17, Anais, (suplemento) $47 \mathrm{p}$.

Beurlen K. 1962. A geologia da Chapada do Araripe. Anais da Academia Brasileira de Ciências, 34(3):365-370.

Beurlen G. \& Regali M.S.P. 1987. O Cretáceo da plataforma continental do Maranhão e Pará, Brasil: biostratigrafia e evolução Paleoambiental. Boletim de Geociências da Petrobras, 1(2):135-155.

Braun O.P.G. 1966. Estratigrafia dos sedimentos da parte 
interior da região Nordeste do Brasil (Bacias do Tucano - Jatobá, Mirandiba e Araripe), Rio de Janeiro. Boletim do DGM/DNPM, 236.

Carvalho M.A. 2001. Paleoenviromental Reconstruction Based on Palynological and Palynofacies Analyses of the Aptian-Albian Succession in the Sergipe Basin, Northeastern Brazil. Tese de Doutoramento, Naturwissenschaftlich-Mathematischen Gesamtfakultät, Ruprecht-Karls-Universität, Heidelberg, 192 p.

Castro J.C., Valença L.M.M., Neumann V.H. 2006. Ciclos e sequências deposicionais das formações Rio da Batateira e Santana (Andar Alagoas), Bacia do Araripe, Brasil. Geociências, 25(3):289-296.

Coimbra J.C., Arai M., Carreño A.L. 2002. Biostratigraphy of Lower Cretaceous microfossils from the Araripe basin, northeastern Brazil. Geobios, 35(6):647-767.

Dino R. 1992. Palinologia, Bioestratigrafia e Paleoecologia da Formação Alagamar - Cretáceo da Bacia Potiguar, Nordeste do Brasil. Tese de Doutoramento, Programa de Pós-graduação em Geologia Sedimentar, Instituto de Geociências, Universidade de São Paulo, São Paulo, 357 p.

Gaspary J. \& Anjos N.F.R. 1964. Estudo Hidrogeológico de Juazeiro do Norte - Ceará. DRN/SUDENE, Série Hidrogeologia, 3, $25 \mathrm{p}$.

Hashimoto A.T., Appi C.J., Soldan A.L., Cerqueira J.R. 1987. O neo-Alagoas nas Bacias do Ceara, Araripe e Potiguar (Brasil); caracterização estratigráfica e paleoambiental. Revista Brasileira de Geociências, 17(2):118-122.

Lima M.R. 1978. Palinologia da Fm. Santana (Cretáceo do Nordeste do Brasil). Tese de Doutoramento, Instituto de Geociências, Universidade de São Paulo, São Paulo, 392 p, +anexos.

Lima M.R. \& Perinotto J.A.J. 1985. Palinologia de sedimentos da parte superior da Formação Missão Velha, Cretáceo do Nordeste do Brasil. Anais da Academia Brasileira de Ciências, 57(1):117-118.

Lima M.R. \& Perinotto J.A.J. 1984. Palinologia de sedimentos da parte superior da Formação Missão Velha, Bacia do Araripe. Geociências, 3:67-76.

Mabesoone J.M \& Tinoco I.M. 1973. Paleoecology of the Aptian Santana Formation (Northeastern Brazil). Palaeogeography, Palaeclimatology, Palaeoecology, 14(2):97-118.

Martill D.M. \& Wilby P. 1993. Stratigraphy. In: Martill D.M.(Ed.). Fossils of the Santana and Crato Formations, Brazil. The Palaeontological Association Field Guides to Fossils, 5:20-50.

Matos R.M.D. 1992. The Northeast Brazilian rift system. Tectonics, 11(4):766-791.

Moraes J.F.S., Scheid C., Santos J.S.A. 1976. Projeto Santana: Relatório Final da Etapa I. Recife, DNPM/ CPRM, 8 v.

Moura M.V. 2007. Análise Estratigráfica do Andar Alagoas na Borda Leste da Bacia do Araripe. Dissertação de Mestrado, Programa de Pós-graduação em Geologia,
Instituto de Geociências, Universidade Federal do Rio de Janeiro, Rio de Janeiro, 108 p.

Neumann V.H. \& Cabrera L. 1999. Una nueva propuesta estratigrafica para la tectonosecuencia post-rift de la Cuenca de Araripe, Noreste de Brasil. In: Simpósio sobre o Cretáceo do Brasil, 5 / Simposio sobre el Cretacico de America del Sur, 1, Boletim, p. 279-285.

Oliveira A.D., Mendonça-Filho J.G., Carvalho M.A., Menezes T.R., Lana C.C., Brenner W.W. 2004. Novo método de preparação palinológicas para aumentar a recuperação de dinoflagelados. Revista Brasileira de Paleontologia, 7(2):169-175.

Paula-Freitas A.B.L. 2010. Análise Estratigráfica do Intervalo Siliciclástico Aptiano da Bacia do Araripe (Formação Rio da Batateira). Dissertação de Mestrado, Programa de Pós-Graduação em Geologia, Instituto de Geociências, Universidade Federal do Rio de Janeiro, Rio de Janeiro, 9 folhas, anexos.

Paula-Freitas A.B.L. 2009. Caracterização Faciológica e Estratigráfica da Formação Rio da Batateira (Andar Alagoas), Bacia do Araripe. Monografia de Graduação, Departamento de Geologia, Instituto de Geociências, Universidade Federal do Rio de Janeiro, Rio de Janeiro, 74 p.

Ponte F.C. \& Appi C.J. 1990. Proposta de revisão da coluna litoestratigráfica da Bacia do Araripe. In: SBG, Congresso Brasileiro de Geologia, 36, Anais, $1: 211-226$.

Ponte F.C. \& Ponte-Filho F.C. 1996. Estrutura Geológica e Evolução Tectônica da Bacia do Araripe. Depto. Nacional de Produção Mineral/MME, Rio de Janeiro, Brasil, 68 p.

Portela H.A. 2008. Estudo Palinológico e Palinofaciológico da Formação Santana, Bacia do Araripe, Nordeste do Brasil. Dissertação de Mestrado, Faculdade de Geologia, Universidade do Estado do Rio de Janeiro, Rio de Janeiro, $133 \mathrm{p}$.

Regali M.S.P. 2001. Palinoestratigrafia dos sedimentos cretácicos da bacias do Araripe e das bacias interiores do Nordeste - Brasil. In: Barros L.M., Nuvens P.C., Filgueira J.B.M. 2001. Simpósio sobre a Bacia do Araripe e Bacias Interiores do Nordeste, 1 e 2. Comunicações, p.101-108.

Regali M.S.P. 1990. Biocronoestratigrafia e paleoambiente do eocretáceo das bacias do Araripe e Rio do Peixe, NE - Brasil. In: Simpósio sobre a Bacia do Araripe e Bacias Interiores do Nordeste, 1. Atas, p.163-171.

Regali M.S.P. 1989. Complicatisaccus cearensis: uma palinozona no Eocretáceo do Brasil. In: Congresso Brasileiro de Paleontologia, 11. Anais, Sociedade Brasileira de Paleontologia, Rio de Janeiro, v.1, p. 235274.

Regali M.S.P. \& Santos P.R.S. 1999. Palinoestratigrafia e geocronologia dos sedimentos albo-aptianos das Bacias de Sergipe e Alagoas - Brasil. In: Simpósio sobre o Cretáceo do Brasil, 5/Simposio sobre el Cretacico de America del Sur, 1, Boletim, p. 411-419.

Regali M.S.P., Uesugui N., Santos A.S. 1974. Palinologia 
dos sedimentos meso-cenozóicos do Brasil (I). Boletim Técnico da Petrobras, Rio de Janeiro, 17(3):177-191.

Rossetti D.F., Góes A.M., Arai M. 2001. A passagem Aptiano - Albiano na Bacia do Grajaú, MA. In: Rossetti, D.F.; Góes, A.M. \& Truckenbrodt (eds.). 2001. O Cretáceo na Bacia de São Luís-Grajaú. Museu Paranaense Emílio Goeldi, p.101-117.

Schaller H. 1969. Revisão estratigráfica da Bacia Sergipe/ Alagoas. Boletim Técnico da Petrobrás, 12(1):21-86.

Scheid C., Munis M.B., Paulino J. 1978. Projeto Santana: Relatório Final da Etapa II. Recife, DNPM/CPRM. $131 \mathrm{p}+$ ilust. e anexos.

Silva M.A.M. 1986. Lower Cretaceous sedimentary sequences in the Araripe Basin, northeastern Brazil: a revision. Revista Brasileira de Geociências, 16(3):311-319.

Silva-Santos R. \& Valença J.G. 1968. A Formação Santana e sua paleoictiofauna. Anais da Academia Brasileira de Ciências, 40(3):339-360.
Small H.L. 1923. Geologia e Suprimento d'Água Subterrânea no Ceará e Parte do Piauhy. Série I.D. Geologia, Publicação n. 25. Inspetoria de Obras Contra as Secas, 2a ed., 80 p, consultada a edição fac-similar publicada na Coleção Mossoroense, 88, de 1979.

Syrio V.N. \& Rios-Netto A.M. 2004. Estudo Bioestratigráfico e Paleoambiental Preliminar de Ostracodes da Formação Rio da Batateira, Bacia Sedimentar do Araripe - Brasil. In: Simpósio sobre o Cretáceo do Brasil, 6/ Simpósio sobre el Cretácico de América del Sur, 2. 2002. Boletim, v.1, p.67-70.

Syrio V.N. \& Rios-Netto A.M. 2002. Ostracodes from the Rio da Batateira Formation (Lower Cretaceous, Araripe Basin): preliminary results on Systematics, Bioestratigraphy and Paleoecology. Anais da Academia Brasileira de Ciências, 74(2):369.

Manuscrito ID 21003

Recebido em: 14/03/2011 Aprovado em: 07/03/2012 
Anexo 1 - Distribuição dos táxons de palinomorfos no poço 1-PS-11-CE, ordenados pelos níveis de último aparecimento.

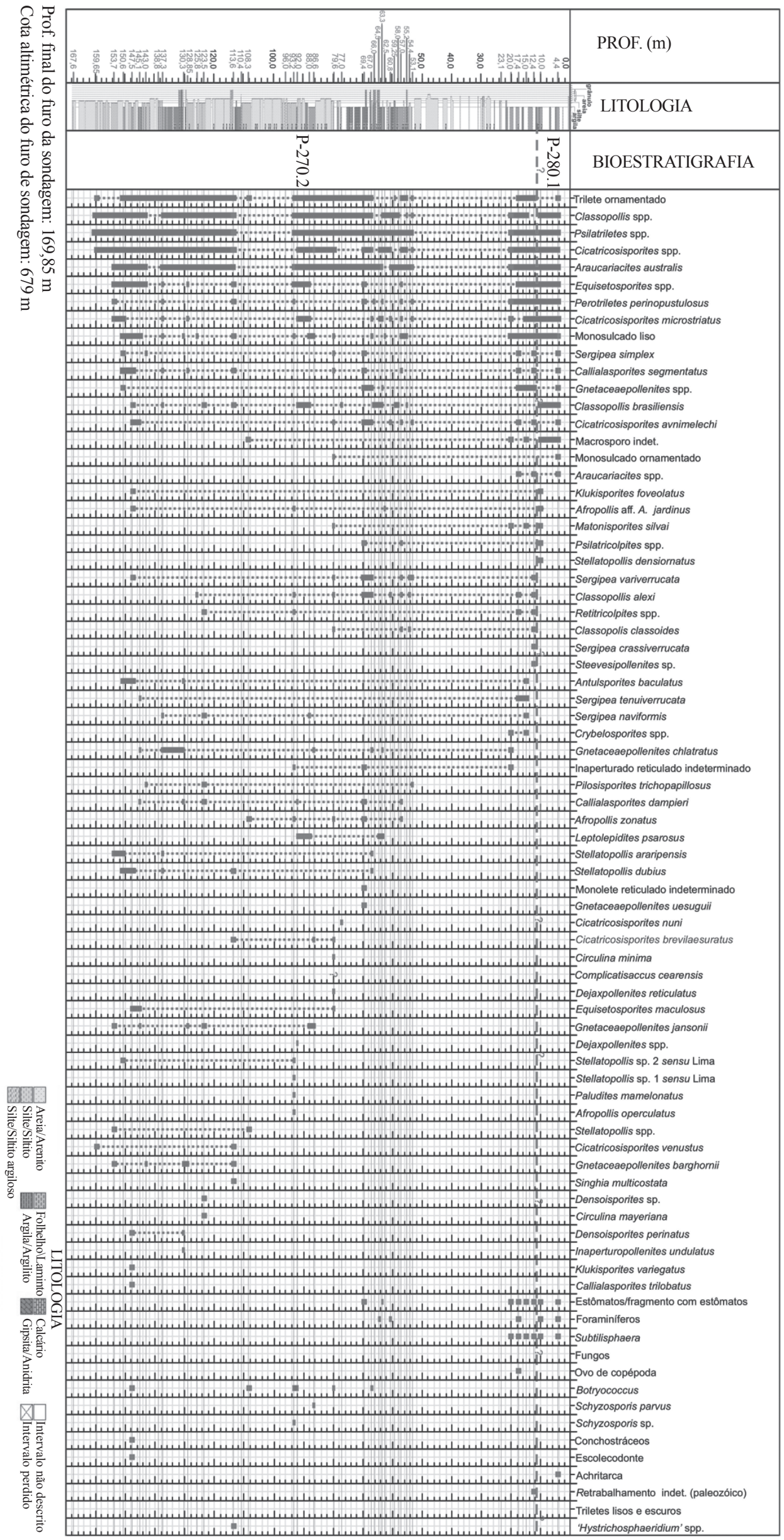


Anexo 2 - Distribuição dos táxons de palinomorfos no poço 1-PS-12-CE, ordenados pelos níveis de último aparecimento.

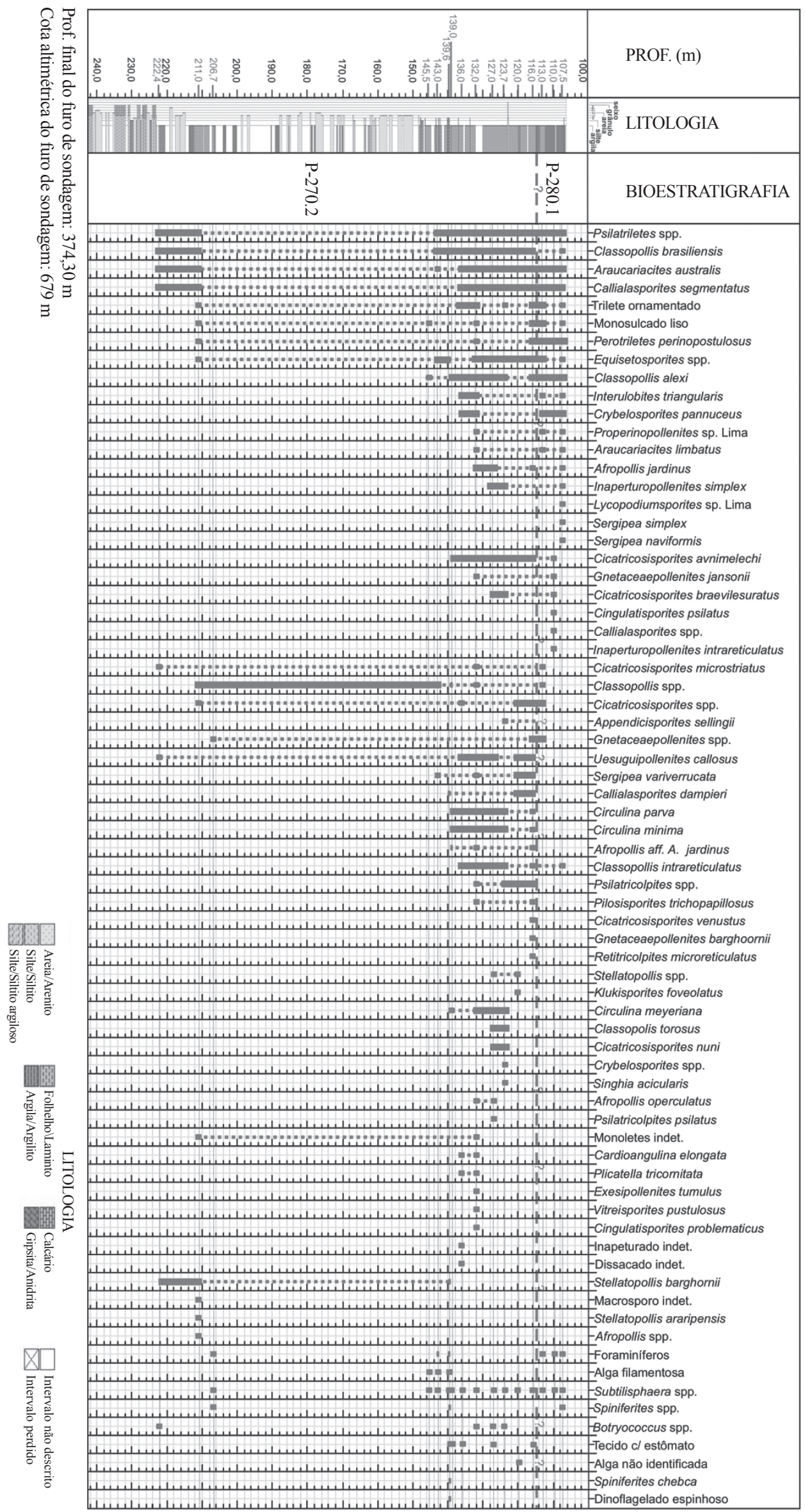

